\title{
Ultra-lean hydrogen-enriched oscillating flames behind a heat conducting bluff-body: anomalous and normal blow-off.
}

\author{
Carmen Jiménez ${ }^{\mathrm{a}, *}$, Dan Michaels ${ }^{\mathrm{b}}$, Ahmed F. Ghoniem ${ }^{\mathrm{c}}$ \\ ${ }^{a}$ CIEMAT, Avenida Complutense 40, 28040 Madrid, Spain \\ ${ }^{b}$ Technion-Israel Institute of Technology, Haifa 32000, Israel \\ ${ }^{c}$ Massachusetts Institute of Technology, 77 Massachusetts Avenue, Cambridge, MA \\ 02139, USA
}

\begin{abstract}
This paper presents a numerical study of ultra-lean hydrogen-methane flames stabilized behind a rectangular, highly conducting metallic bluff body acting as a flame holder. Using high fidelity numerical simulations, we show that lean inverted steady flames exist below normal flammability limits. They have distinct stabilization mechanism from pure methane flames. These flames are blown-off for sufficiently small velocities, a phenomenon called anomalous blow-off. At even leaner conditions oscillating ultra-lean hydrogenmethane flames can be established. These oscillating flames exist within a rather small range of equivalence ratios and inflow velocities, and move to mean locations closer to the flame holder as the reactant flow is increased. We show that the oscillations are associated with the shedding of flame balls from the downstream end of a "residual flame" that remains attached. Unlike their steady counterparts, the oscillating flames exhibit blow-off at both
\end{abstract}

\footnotetext{
*Corresponding author:

Email address: carmen.jimenez@ciemat.es (Carmen Jiménez )
} 
low velocities (anomalous blow-off) and at sufficiently high inflow velocities (normal blow-off). We show that normal blow-off is linked to heat losses to the flame holder.

Keywords:

laminar flames, bluff-body stabilization, ultra-lean, conjugate heat exchange, hydrogen 


\section{Introduction}

One of the practical means of effectively obtaining a stable premixed flame in a high velocity reactant flow over a wide range of inflow rates is using a solid obstacle as a flame holder. The solid body immersed in the flow creates a low velocity recirculation zone where the flame can anchor even when the burning velocity is very small compared to the mean flow velocity. The recirculating flow produces a flux of hot products towards the reactants that contribute to their preheating, therefore favoring ignition conditions. Additionally, the solid body can contribute to flame stabilization by providing means by which some of the heat produced in the combustion zone is conducted through the solid back to preheat the reactants. Aerodynamic strain and curvature, together with possible effects of differential diffusion and heat transport determine the local flame propagation speed and its shape.

Starting with the pioneering work of Lewis and von Elbe [1, 2], who performed experiments of inverted flames stabilized behind a central body and linked the blow-off to the velocity gradient at the nozzle reaching a critical value, many researchers have tried to explain the mechanisms that affect flame stabilization and blow-off in these flames, adding other factors such as flame curvature $[3,4]$ and heat exchange with the flame holder [3-6]. Numerical studies where the combustion field, its heat exchange with the solid body and the internal temperature distribution inside the latter can be coupled have been scarce until recent years. Advances in computational power and parallel computing as well as specific coupling strategies $[7,8]$ have allowed direct numerical simulations of this kind of problems using detailed chemistry and transport properties of complex fuels and including 
the conjugate heat exchange with the solid [9-14].

A recent experimental study [15] examined methane-air and methanehydrogen-air flames anchored behind a long, highly conducting metallic cylindrical rod with a small diameter (1 to $3 \mathrm{~mm}$ ), placed along the axis of a circular channel. The study showed that in this configuration hydrogen-containing flames could be stabilized for mixtures well below the flammability limit, permitting ultra-lean combustion. It showed that pure methane flames behave according to the well-know mechanism, anchoring farther away from the solid rod as the flow rate is raised and eventually blowing off at a high enough flow rate. When the burning mixture contains a sufficient amount of hydrogen, this behavior is different: increasing the flow rate results in the flame anchoring closer to the metallic rod and reducing the flow rate leads to flames stabilized farther from the flame holder and can eventually lead to blow-off. This blow-off by decreasing the inflow velocity, termed "anomalous blow-off" in [15], was found when hydrogen content was equal or larger than $20 \%$. Dual blow-off was also reported for some of these flames: "anomalous" blow-off by decreasing the inflow velocity and "normal" blow-off as the flow velocity is raised.

In a previous study [16], we used direct numerical simulations to investigate the anchoring of ultra-lean $\mathrm{H}_{2}-\mathrm{CH}_{4}$ flames behind a thin conducting solid. We reproduced the experimental findings, using a 2D planar model, with a rectangular metallic bluff body of size 5 x $15 \mathrm{~mm}$. For $40 \% \mathrm{H}_{2}-60 \% \mathrm{CH}_{4}$ mixtures, it was found, as in the experiments, that flames could be established for mixtures under the lean flammability limit of the unstrained planar flame. For these ultra-lean flames, as the inflow reactants velocity increased, 
the flame stabilized closer to the flame holder. Conversely, as the inflow velocity was reduced, the steady flame moved farther away from the flame holder, and for a sufficiently small value of this velocity the flame was blownoff. We showed in [16] that this anomalous stabillzation behavior was related to the high diffusivity of $\mathrm{H}_{2}$, which results in $\mathrm{H}_{2}$ concentrating on the highly strained and curved flame base, and leading to local burning at richer equivalence ratios. As the inflow rate is increased, flame stretch near the flame base is increased and this focusing effect is enhanced, leading to stronger burning at the flame base and it moving closer to the flame holder, anchoring inside the flow recirculation zone. When the inflow rate is reduced below a certain limit there is not enough stretch at the flame base to generate a locally richer mixture region and the flame is extinguished.

However, the simulations in [16] did not show blow-off as the inflow reactants velocity was increased. Raising the velocity resulted always in steady flames stabilizing closer to the solid. Thus "normal blow-off", as reported in the experiments, was not reproduced in our numerical investigation.

In this paper, we report new results at even leaner equivalence ratios in the same configuration studied in [16]. We show that, as the equivalence ratio is reduced, unsteady flames featuring oscillatory dynamics may appear. These very weak flames exhibit both "anomalous" and "normal" blow-off. As in [16], we use direct numerical simulations including detailed chemistry and species transport, as well as the conjugate heat exchange with the conducting body in a planar (2D) configuration. 


\section{Numerical model and simulation set-up}

Under the low-Mach number approximation, the conservation equations for mass, momentum, energy and species are:

$$
\begin{aligned}
\frac{\partial \rho}{\partial t}= & -\nabla \cdot(\rho \mathbf{v}) \\
\frac{\partial \mathbf{v}}{\partial t}= & -\frac{1}{\rho} \nabla p-(\mathbf{v} \cdot \nabla) \mathbf{v}+\frac{1}{\rho} \nabla \cdot \tau, \\
\frac{\partial T}{\partial t}= & -(\mathbf{v} \cdot \nabla) T+\frac{1}{\rho c_{p}} \nabla \cdot(\lambda \nabla T)-\left(\sum_{k=1}^{N_{s}} c_{p, k} Y_{k} \mathbf{V}_{k}\right) \cdot \nabla T \\
& -\frac{1}{\rho c_{p}} \sum_{k=1}^{N_{s}} h_{k} \dot{\omega}_{k}, \\
\frac{\partial Y_{k}}{\partial t}= & -(\mathbf{v} \cdot \nabla) Y_{k}-\frac{1}{\rho} \nabla \cdot\left(\rho Y_{k} \mathbf{V}_{k}\right)+\frac{1}{\rho} \dot{\omega}_{k}, k=1, \ldots, N_{s} .
\end{aligned}
$$

where $\mathbf{v}$ is the velocity vector, $\rho$ the density, $T$ the temperature, $Y_{k}$ the $k$ species mass fraction, $p$ the hydrodynamic pressure and $N_{s}$ the number of involved chemical species. This system of equations is supplemented with the equation of state for an ideal gas: $p_{0}=\frac{\rho \mathcal{R} T}{\bar{W}}$, where $p_{0}$ is the thermodynamic pressure, considered spatially uniform in the low-Mach number limit, and also constant in time in the present, open-domain configuration; $\mathcal{R}$ stands for the universal gas constant, and $\bar{W}$ corresponds to the mixture molecular weight.

In Eqs. 1, $\tau$ is the stress tensor of components: $\tau_{i j}=\mu\left(\frac{\partial u_{i}}{\partial x_{j}}+\frac{\partial u_{j}}{\partial x_{i}}-\frac{2}{3} \delta_{i j} \nabla \cdot \mathbf{v}\right)$, with $\mu$ the dynamic viscosity of the mixture and $u_{i}$ the components of the velocity vector; $\mathbf{V}_{k}$ is the diffusion velocity of species $k$, modeled as: $\mathbf{V}_{k}=-\frac{D_{k, m}}{X_{k}}\left(\nabla X_{k}\right)-\frac{D_{k}^{T}}{\rho Y_{k}} \frac{\nabla T}{T}$, where $X_{k}$ stands for the mole fraction of species $k, D_{k, m}$ is the mixture-averaged diffusivity of species $k$ and $D_{k}^{T}$ is its thermal diffusion coefficient, which is introduced to account for the Soret effect. 


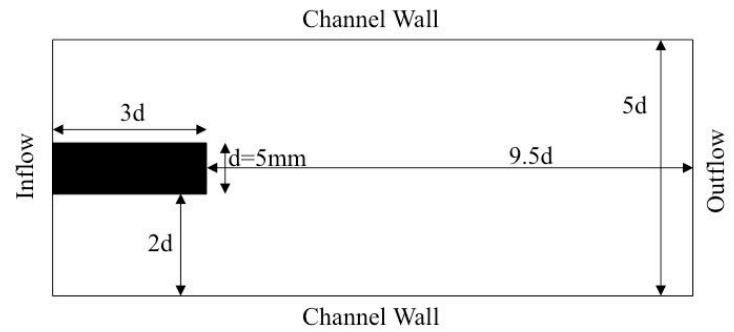

Figure 1: A sketch of the computational domain.

Moreover, $c_{p, k}$ and $c_{p}$ stand for the specific heat at constant pressure for species $k$ and for the mixture, resp., $\lambda$ is the mixture thermal conductivity and $h_{k}$ and $\dot{\omega}_{k}$ represent the molar enthalpy and molar production rate (per unit time and volume) of species $k$. The NASA polynomials [17] are used to compute the thermodynamic properties of the mixture; the mixture-averaged transport properties are evaluated using a dipole-reduced formalism [18].

The conjugate heat exchange between the solid and the fluid is incorporated by simultaneously integrating the conservation equations for the reacting flow, Eqs. 1, and the transient heat conduction equation in the solid body:

$$
\frac{\partial T}{\partial t}=\frac{1}{\rho_{s} c_{p s}} \nabla \cdot\left(\lambda_{s} \nabla T\right),
$$

where $\rho_{s}, c_{p s}$ and $\lambda_{s}$ are, resp., the density, the heat capacity and the thermal conductivity of the solid material. A no-penetration condition, the continuity of the temperature field and the conservation of heat flux are imposed at each time step in the solid-fluid boundary using the buffer-zone method presented in [19]. Numerical integration of Eqs. 1 is performed using the adaptive mesh refinement method proposed in [20]. 
Figure 1 presents a schematic of the geometry of the computational domain. The flame holder, shaded in black, is a rectangular body of width $d=5 \mathrm{~mm}$ and length $l=3 d$. The computational domain is a channel of height $h=5 d$ and length $L=12.5 d$. The flame holder is considered to be a highly conducting metal with $\lambda_{s}=60 \mathrm{~W} /(\mathrm{m} \mathrm{K})$ and $\rho_{s} c_{p s}=56532 \mathrm{~J} /\left(\mathrm{m}^{3}\right.$ $\mathrm{K})$.

The fuel composition is $40 \% \mathrm{H}_{2}$ and $60 \% \mathrm{CH}_{4}$ by volume. For this fraction of hydrogen, ultra-lean combustion and anomalous blow-off were reported in the experiments in [15]. The fuel is premixed with air to create mixtures at equivalence ratios $\phi$ between 0.3 and 0.4 ( $\phi$ is defined according to the stoichiometric relation: $\phi=\frac{1}{2} \frac{X_{\mathrm{H}_{2}}}{X_{\mathrm{O}_{2}}}+2 \frac{X_{\mathrm{CH}_{4}}}{X_{\mathrm{O}_{2}}}$.) The skeletal chemical kinetics scheme proposed in [21], consisting of 46 reversible reactions among 16 species is used to compute the species production rates. This mechanism has been validated for lean $\mathrm{CH}_{4} / \mathrm{H}_{2}$ flames in [13]. The lean flammability limit of a $40 \% \mathrm{H}_{2}-60 \% \mathrm{CH}_{4}$ planar unstrained flame using this chemical kinetics is $\phi=0.425$, and hence all the mixtures in the present study are below this flammability limit.

Boundary conditions at the inflow of the computational domain correspond to a fully developed Poiseuille flow above and below the solid body with mean velocity $U$, reactant mass fractions given by $\phi$ and pressure and temperature $p_{0}=1 \mathrm{~atm}, T_{0}=300 \mathrm{~K}$, so that the total mass flux at the inlet is $\dot{m}=\rho_{0} U 4 d$. $U$ is varied between $5 \mathrm{~cm} / \mathrm{s}$ and $8.5 \mathrm{~m} / \mathrm{s}$, resulting in a Reynolds number variation from $R e_{d}=15$ to $R e_{d}=2590$, based on the flame holder width $d$. At the outlet, "convective-flow" boundary conditions are used for the velocity and scalars and a Neumann (zero-gradient) 
condition is applied to the pressure [20]. No-slip, impermeable, adiabatic conditions are imposed at the channel walls, while the left wall of the solid body was kept isothermal, at ambient temperature equal to $T_{0}=300 \mathrm{~K}$. This boundary condition models cooling of the body at a distance of $15 \mathrm{~mm}$ from the flame side. This distance was shown in [16] to have no significant effect on the flame stabilization location or solid body temperature field for the present value of the thermal conductivity.

The size of the computational grid was $\Delta x=\Delta y=196 \mu \mathrm{m}$, with one additional refinement level in the adaptive mesh refinement. This mesh resolution results in nearly 15 points inside the flame thickness of a planar flame near the flammability limit $\phi=0.425$, which is estimated to be $\delta_{T}=\lambda /\left(\rho c_{p} S_{L}\right) \approx 1.5$ $\mathrm{mm}[16]$. Given that the ultra-lean flames below the lean flammability limit of the present study are slower and consequently thicker, the same level of resolution should also suffice.

\section{Steady flame solutions}

Examples of steady flame solutions, corresponding to reactant mixtures with $40 \% \mathrm{H}_{2}-60 \% \mathrm{CH}_{4}$ at an equivalence ratio $\phi=0.35$ and inflow velocities $U=0.5$ and $U=1.5 \mathrm{~m} / \mathrm{s}$, and at equivalence ratio $\phi=0.325$ and inflow velocity $U=1.5 \mathrm{~m} / \mathrm{s}$ are presented in Fig. 2. In this figure the flow goes from left to right and the flames are represented by colored contours of the temperature field while black solid lines mark the isosurfaces where the heat release rate is equal to $10 \%$ and to $50 \%$ of the maximum value. Selected streamlines are added to locate the recirculation zone. Figure 2 shows that, as the inflow velocity is raised, the flame moves closer to the flame holder, 

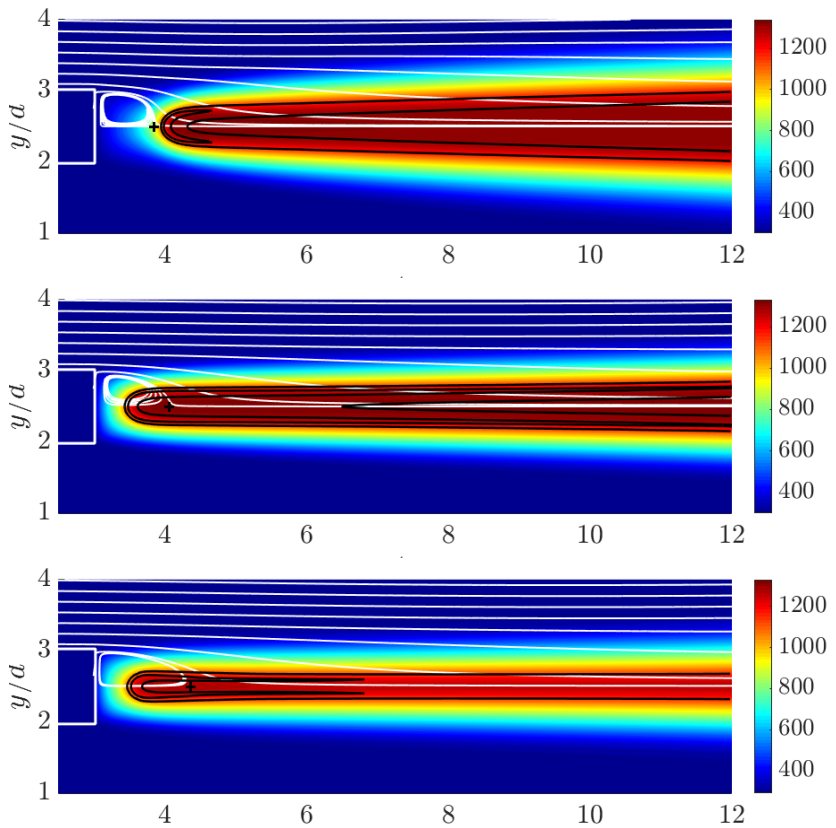

Figure 2: Temperature field for steady flames corresponding to $40 \% \mathrm{H}_{2}-60 \% \mathrm{CH}_{4}$ at different equivalence ratios $\phi$ and inflow velocities $U$ : (top) $\phi=0.35, U=0.5 \mathrm{~m} / \mathrm{s}$; (middle) $\phi=0.35, U=1.5 \mathrm{~m} / \mathrm{s}$; (bottom) $\phi=0.325, U=1.5 \mathrm{~m} / \mathrm{s}$. The black lines mark the surfaces where the heat release is $10 \%$ and $50 \%$ of the maximum. The white lines represent selected streamlines and the + sign marks the end of the recirculation zone. 


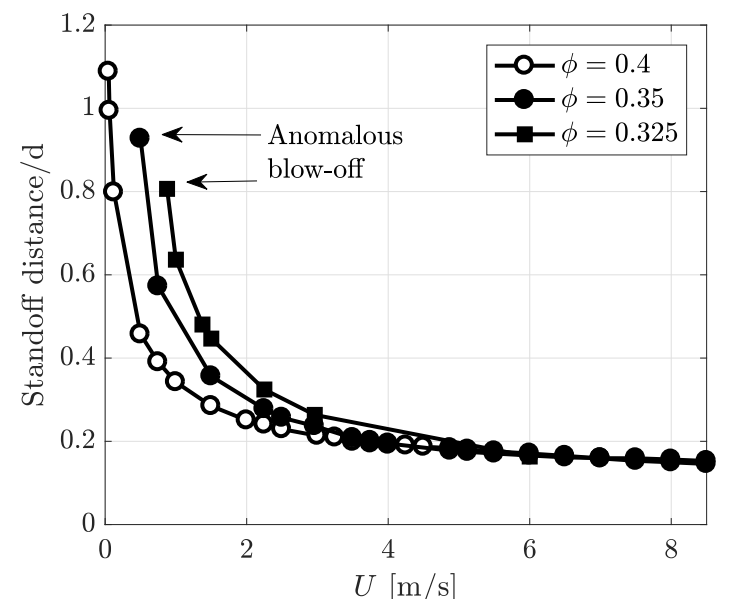

Figure 3: The stand-off distance (distance from the flame base to the flame holder) for flames with $40 \% \mathrm{H}_{2}-60 \% \mathrm{CH}_{4}$ at equivalence ratios $\phi=0.4,0.35$ and 0.325 as a function of the reactants inflow velocity $U$.

anchoring inside the recirculation zone. Moreover, as the mixture equivalence ratio is lowered, the flame becomes parallel to the flow and moves slightly away from the flame holder.

The flame response to both independent parameters can be seen in Fig. 3, which presents the stand-off distance, or distance from the flame base to the solid, for flames at $\phi=0.4,0.35$ and 0.325 over a wide range of $U$. All the results in Fig. 3 correspond to steady symmetric flames. The exact value of the stand-off distance depends on the definition of the flame surface; here it is defined as the location where the heat release rate is $10 \%$ of the maximum heat release.

It is clear from Fig. 3 that for these flames, as the reactant mass flow rate is raised, the flame anchors at a point closer to the flame holder. In the case at equivalence ratio $\phi=0.4$ the inflow velocity can be reduced to very small 
values and still a steady flame exists. For $\phi=0.35$ the minimum velocity for which a steady flame exists is $U=0.5 \mathrm{~m} / \mathrm{s}$; reducing this velocity results in flame blow-off. For $\phi=0.325$, the minimum velocity before blow-off is $U=0.85 \mathrm{~m} / \mathrm{s}$. Therefore for $\phi=0.35$ and $\phi=0.325$ our results show the behavior termed as anomalous blow-off in [15]: the anchoring distance decreases as the inflow velocity is raised and increases as this velocity is reduced, so that for a certain low value of the inflow velocity the flame can not be sustained.

For the flames represented in Fig. 3, as $U$ is raised the flames move closer to the flame holder, but no extinction or blow-off are detected, not even for values of the inflow velocity as high as $U=8.5 \mathrm{~m} / \mathrm{s}$. In the experiments in [15], blow-off was observed for a narrow range of equivalence ratios when the inflow velocity was raised, at values between $U=2$ and $4 \mathrm{~m} / \mathrm{s}$; this is what the authors called normal blow-off. No explanation was found in our study in [16] for this different behavior at high velocities. The following results show a possible explanation.

\section{Unsteady flames}

Starting from a steady flame burning a $40 \% \mathrm{H}_{2}-60 \% \mathrm{CH}_{4}$ mixture at an inflow rate $U=1.5 \mathrm{~m} / \mathrm{s}$, if the equivalence ratio is reduced from $\phi=0.35$ to $\phi=0.325$ (as in the flames shown in Fig. 2), the total heat release rate in the flame is reduced. As the equivalence ratio is lowered further, oscillating flame solutions emerge. Figure 4 shows a sequence of snapshots of the temperature and heat release rate in an oscillating flame with $\phi=0.315$ and $U=1.5$ $\mathrm{m} / \mathrm{s}$, at times $0.003 \mathrm{~s}$ apart. The oscillations are driven by periodic pinch-off 
events, similar to the pinch-off events leading to blow-off described in [11]. For the present flames, pinch-off leads to blow-off if the equivalence ratio is lowered below about $\phi=0.3$, but for intermediate values of $\phi$ unsteady flames survive. Because of the low equivalence ratio of the mixture, burning occurs mainly in mostly curved end sections of the flame, as can be seen in Fig. 4 (right). For the flame that remains attached to the bluff body, which we refer to as the residual flame, two intensely burning curved regions at the base (upstream) and the tip (downstream) are observed. The flame consumes reactants from all sides and its tip moves downstream, as a consequence the residual flame length increases. The flame base is stabilized near the solid wall. As the residual flame length grows, there are regions where it becomes flat and almost parallel to the flow, and its burning velocity reduces leading to a pinch-off event. This occurs in the present cycle near a location $x / d=5$ at a time just after $t=0.003 \mathrm{~s}$, corresponding to the sudden decrease in the residual flame length and heat release observed in Fig. 4. In this pinch-off event the flame is broken in two regions: a flame ball, which burns intensely because of its curvature and is convected downstream, and a smaller residual flame. After pinch-off the residual flame begins to expand downstream again, burning unburnt reactants and the cycle starts again.

The amplitude and frequency of these oscillations change with the mixture equivalence ratio, as shown in Fig. 5. Here we present the instantaneous value of the total heat release rate in the residual flame as $\phi$ is lowered. For $\phi=0.32,0.315$ and 0.314 the heat release rate shows oscillations with frequencies 38,8 and $4 \mathrm{~Hz}$, respectively. As the equivalence ratio is lowered to a leaner value, $\phi=0.313$, the oscillating flame extinguishes. 
Figure 5 also shows that as $\phi$ is reduced the flame power (total heat release in the residual flame) is also appreciably reduced, the flames become weaker. Interestingly, in the simulations these weak, oscillating flames were found to suffer "normal blow-off". As the inflow reactants velocity $U$ is raised their distance to the flame holder is reduced, leading to increase in heat losses. The oscillation frequency decreases with leaning the mixture and also with increasing the flow velocity (see Fig. 6). In both cases this is linked to weaker burning (because of lower $\phi$ or larger heat losses, respectively). With weaker burning the flame elongation after each pinch-off becomes slower, and pinchoff events occur at longer periods.

As heat loss becomes excessive in these weak flames they blow-off. This is illustrated in Fig. 6 for flames with $\phi=0.314$. As $U$ is raised from $U=1.25 \mathrm{~m} / \mathrm{s}$ to $1.5 \mathrm{~m} / \mathrm{s}$ the heat produced in the residual flame decreases only slightly, but, because the flame moves to a position closer to the solid body, the fraction of this heat lost to the solid increases appreciably. As $U$ is raised further to $U=1.65 \mathrm{~m} / \mathrm{s}$ the fraction of heat lost to the flame holder becomes too large and the flame is blown-off.

The map in Fig. 7 shows steady (black circles) and unsteady (white circles) flame solutions as the equivalence ratio and the inflow velocity are varied. Flames exist for values of these two parameters above or on the dashed line; as the line is crossed to lower values of $\phi$ or by varying $U$ at constant $\phi$, blow-off occurs. For the highest values of equivalence ratio in this figure, $\phi>0.32$, blow-off is associated to a decrease in the inflow velocity ("anomalous blow-off"), while steady flames exist for any large value of the inflow velocity. For the lowest equivalence ratios, $\phi \leq 0.32$, two extinction points 
are found, corresponding to decreased and increased velocity $U$. For these flames, which are unsteady, both "anomalous" and "normal" blow-off were found.

These unsteady flame solutions are very plausibly the flames for which "normal blow-off" was detected in the experiments in [15]. Indeed, flame oscillations prior to high velocity blow-off were reported in those experiments, even though they were interpreted as extinction-reignition events [15]. In a recent paper analyzing numerically the same problem it was shown, using linear stability analysis, that inverted flames could be unstable for very lean mixtures, resulting in oscillating flames [22]. The supplemental material included in the online version of [22] shows oscillations very similar to those found here. We note that in [22] a simplified model with constant density was used; the model results showed that these oscillations are related to flame instabilities (and not to hydrodynamic instabilities). Our present results confirm the existence of these instabilities, showing the parametric range at which they are expected and their link to normal blow-off of ultra-lean hydrogen-enriched flames.

\section{Conclusions}

We investigated ultra-lean $\mathrm{H}_{2}-\mathrm{CH}_{4}$ flames behind a thin heat conducting bluff-body. For this purpose, we used direct numerical simulations, including detailed chemistry and species transport, as well as the conjugate heat exchange with the conducting body in a planar (2D) configuration.

Steady flame solutions were found for $40 \% \mathrm{H}_{2}-60 \% \mathrm{CH}_{4}$ mixtures at equivalence ratios below the planar flame lean flammability limit (in the range 
$\phi=0.4-0.325)$. For the leanest cases $(\phi=0.35,0.325)$ "anomalous blowoff" was detected, as in the experiments of [15]: as the inflow reactants velocity is raised, the flame stabilizes closer to the flame holder; conversely, as the inflow velocity is reduced, the steady flame moves farther away from the flame holder, and for a sufficiently small value of this velocity the flame was blown-off. This behavior was described in our previous study in [16], where we showed that it was linked to the presence of hydrogen in the reactants and its large diffusivity.

In the present paper, we have shown that for a certain, rather small, range of very lean equivalence ratios, unsteady flames displaying oscillating dynamics appear. These oscillating flames are weaker than the steady flames obtained at slightly higher equivalence ratios, so that heat losses to the conducting flame holder can become relatively important. Therefore, for these flames, blow-off may occur as a consequence of an increase in the inflow velocity, if heat losses become too high as the flame approaches the solid body. This explains the "normal blow-off", or blow-off at high inflow velocities, that was also reported in the experiments of [15].

\section{Acknowledgments}

This work was supported partly by a MIT-Technion fellowship to D. Michaels, by a KAUST grant number KUS-110-010-01, and by the MINECO/FEDER grant number ENE2015-65852-C2-2-R.

\section{References}

[1] B. Lewis, G. von Elbe, J. Chem. Phys. 11 (1943) 75-97. 
[2] B. Lewis, G. von Elbe, Combustion, Flames and Explosions of Gases, Academic Press, New York, 1961.

[3] T. Kawamura, K. Asato, T. Mazaki, T. Hamaguchi, H. Kayahara, Combust. Flame 35 (1979) 109-116.

[4] T. Kawamura, K. Asato, T. Mazaki, Combust. Flame 45 (1982) 225-233.

[5] C. Sung, C. Law, A. Umemura, Proc. Comb. Inst. 24 (1992) 205-212.

[6] C. Treviño, S. Donnerhack, N. Peters, Combust. Flame 85 (1991) 505510.

[7] F. Duchaine, A. Corpron, L. Pons, V. Moureau, F. Nicoud, T. Poinsot, Int. J. Heat Fluid Flow 30 (2009) 1129-1141.

[8] C. Koren, R. Vicquelin, O. Gicquel, Int. J. Thermal Sci. 118 (Supp. C) (2017) 340-354.

[9] K. S. Kedia, A. F. Ghoniem, Combust. Flame 159 (2012) 1055-1069.

[10] K. S. Kedia, A. F. Ghoniem, Combust. Flame 161 (2014) 2327-2339.

[11] K. S. Kedia, A. F. Ghoniem, Combust. Flame 162 (2015) 1304-1315.

[12] D. Michaels, A. F. Ghoniem, Combust. Flame 172 (2016) 62-78.

[13] D. Michaels, S. Shanbhogue, A. Ghoniem, Combust. Flame 176 (2017) $151-161$.

[14] M. Miguel-Brebion, D. Mejia, P. Xavier, F. Duchaine, B. Bedat, L. Selle, T. Poinsot, Combust. Flame 172 (2016) 153-161. 
[15] Y. Shoshin, R. J. M. Bastiaans, L. P. H. de Goey, Combust. Flame 160 (2013) 565-576.

[16] C. Jiménez, D.Michaels, A.F. Ghoniem, Combust. Flame 191 (2018) $86-98$

[17] B. M. Bride, S. Gordon, M. Reno, Tech.Rep. NASA TM-4513, NASA, 1993.

[18] P. Paul, Tech. Rep.SAND-98-8203, Sandia National Labs, 1997.

[19] K. S. Kedia, C. Safta, J. Ray, H. N. Najm, A. F. Ghoniem, J. Comp. Phys. 272 (2014) 408-428.

[20] C. Safta, J. Ray, H. N. Najm, J. Comp. Phys. 229 (2010) 9299-9322.

[21] M. Smooke, I. Puri, K. Seshadri, Proc. Comb. Inst. 21 (1988) 17831792.

[22] V. Kurdyumov, Y. Shoshin, L. de Goey, Proc. Comb. Inst. 35 (2015) 981-988. 

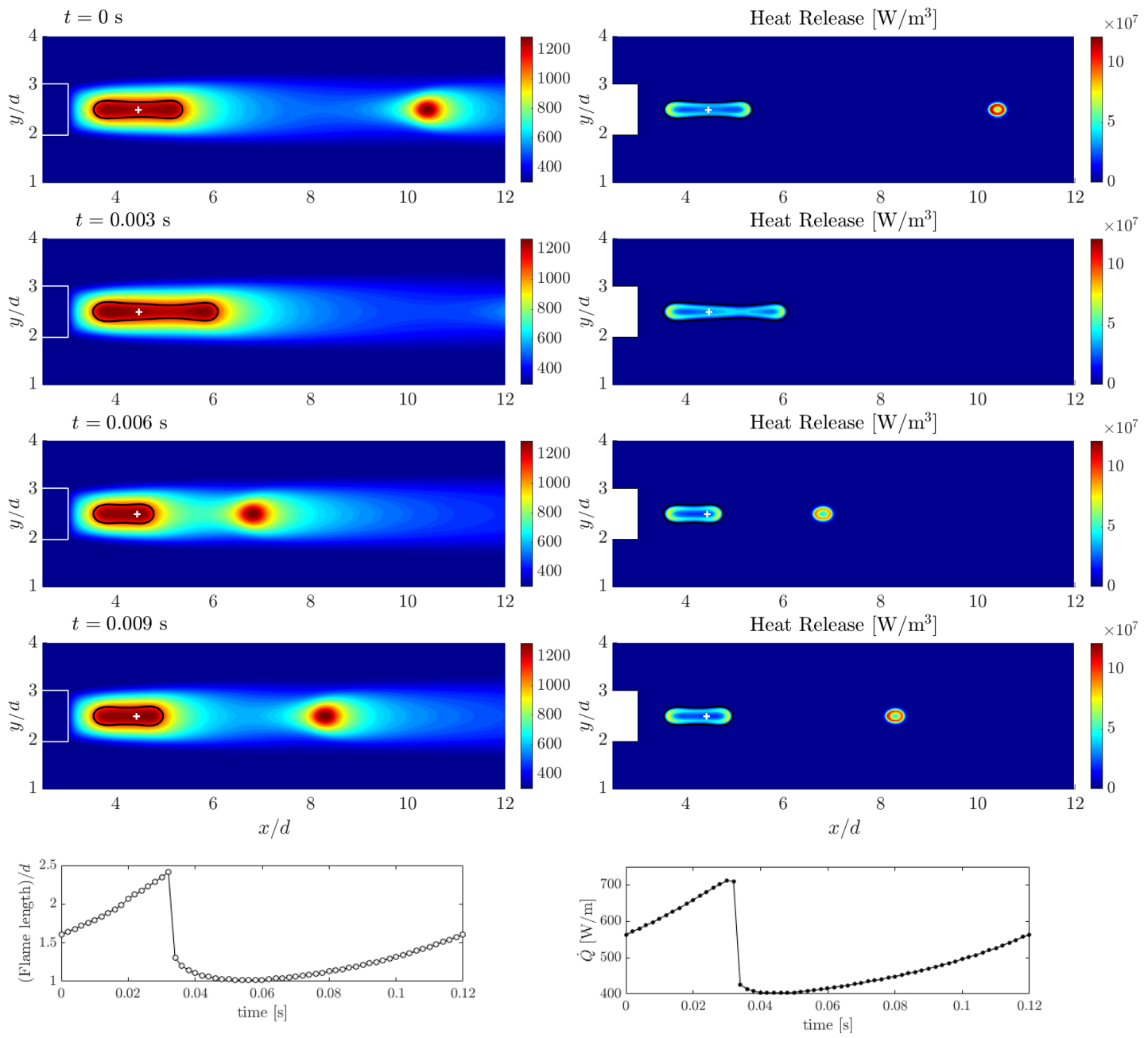

Figure 4: Temperature field (left) and heat release rate (right) in an oscillating flame with $\phi=0.315$ and $U=1.5 \mathrm{~m} / \mathrm{s}$. The black line corresponds to $10 \%$ of the heat release rate and the + marks the recirculation zone end. (Bottom) Residual flame length and heat release rate along the cycle. 


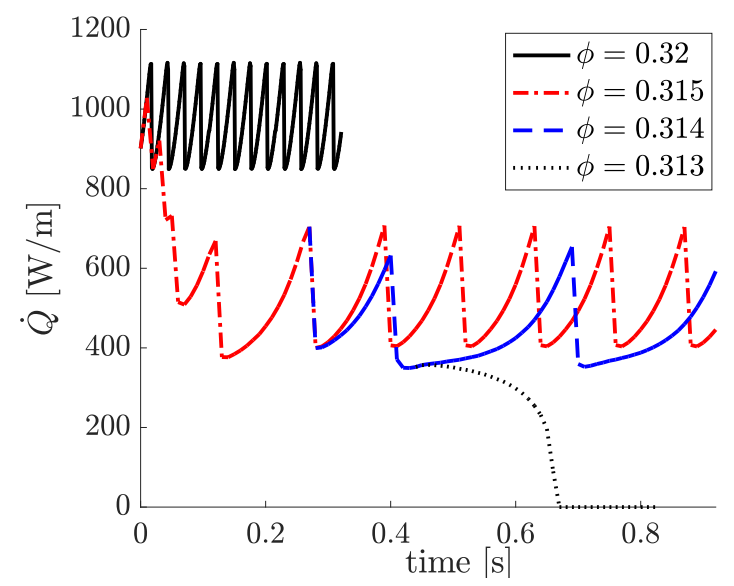

Figure 5: Variation of the total heat release rate in the residual flames with $\phi=0.32$ to $\phi=.313$ at an inflow reactants velocity $U=1.5 \mathrm{~m} / \mathrm{s}$. 

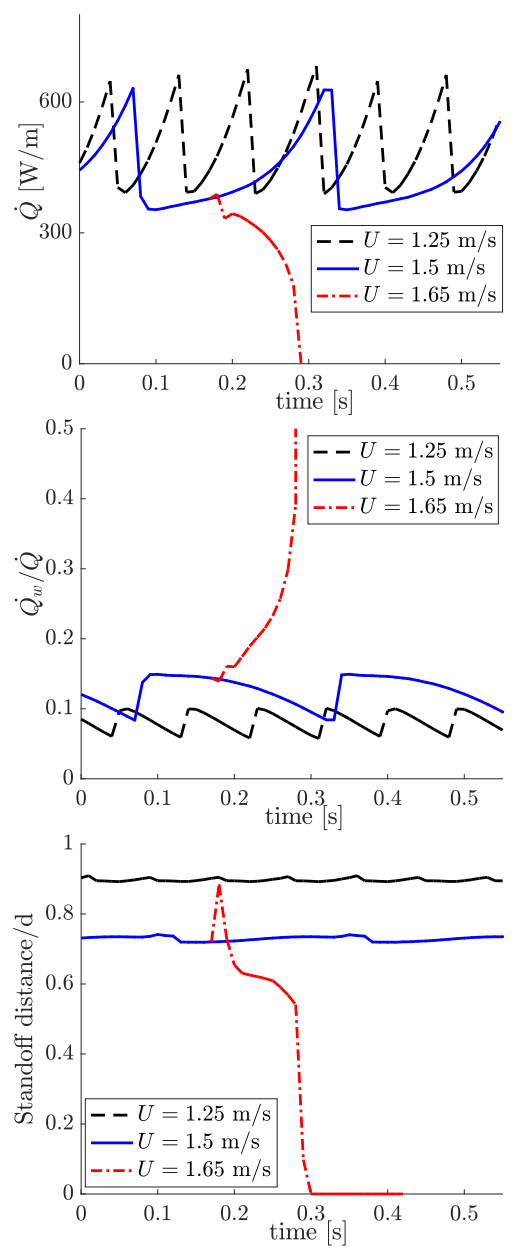

Figure 6: (Top) Total heat release rate in the residual flame, (middle) fraction of this heat lost to the flame holder and (bottom) standoff distance during oscillations in flames with $\phi=0.314$ and varying inflow velocity $U$. 


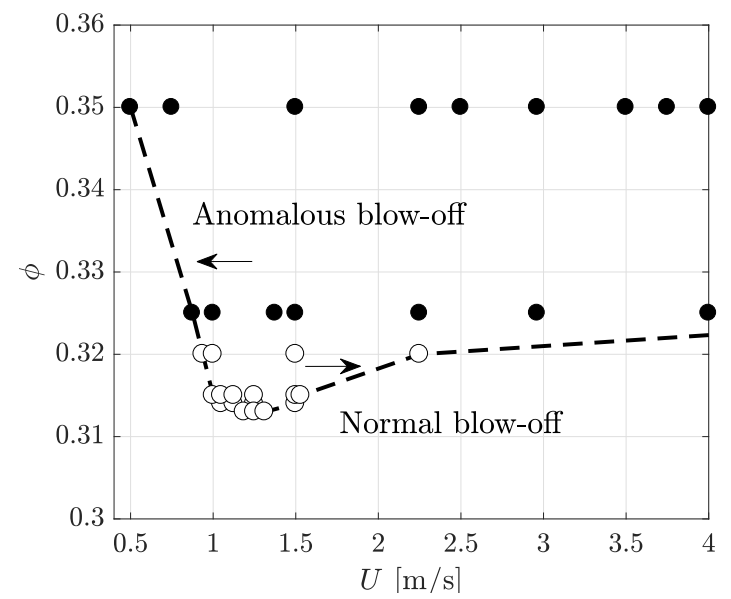

Figure 7: Low and high velocity blow-off points at different equivalence ratio. Flames exist to the top of the dashed line, and are blown-off as the line is crossed in any direction. Black circles correspond to steady flames while white circles represent unsteady solutions. 